\title{
Acute Retinal Necrosis a Potentially Visual Devastating Affection
}

Khanaouchi N*, Khoyali A, Zerrouk R, Mouzarii Y, Reda K and Oubaaz A

Hopital Militaire d'instruction Mohamed V de Rabat, Morocco

*Corresponding author: Nawal Khanaouchi, Hopital Militaire d'instruction Mohamed V de Rabat, Morocco

Received: August 12, 2021; Accepted: September 08, 2021; Published: September 15, 2021

\section{Introduction}

Described firstly in 1971 by Uryama et al. the acute retinal necrosis syndrome (ARN syndrome) is a rare condition, caused by DNA viruses mainly Varicella Zoster Virus (VZV) and Herpes simplex virus type (1 and 2), Cytomegalovirus (CMV) is less common, the diagnosis is mainly based on the suggestive clinical appearance. The institution of antiviral therapy should not be delayed. Prompt diagnosis and treatment are crucial to salvage vision in this frequently blinding condition and preventing involvement of the fellow eye.

The prognosis remains poor due to the extension of retinal necrosis and the risk of retinal detachment in an area of retinal atrophy.

\section{Case Presentation}

We report the case of a 56-year-old women, diabetic, who presented to the emergency room for rapidly progressive loss of vision of her left eye, associated with redness and ocular pain evolving, for 10 days with worsening of symptoms since 3 days.

The visual acuity at admission was at counting fingers; slit lamp examination showed granulomatous retro-desmetic opacities with +3 cells in the anterior chamber, IOP at $18 \mathrm{~mm} \mathrm{Hg}$ in the OS.

The fundus examination of the left eye, revealed severe vitritis, associated with necrotic-hemorrhagic patches, and cystoid vasculitis

\section{Abstract}

Acute retinal necrosis syndrome (ARN syndrome) is a rare condition, caused by DNA viruses. The diagnosis of this condition is mainly based on the uggestive clinical appearance. Prompt diagnosis and treatment is crucial to prevent visual loss. We report the case of 56-year-old women, diabetic, who sented to the emergency room for rapid decrease of visual function of her left e, which revealed granulomatous uveitis with retinal necrosis.

Keywords: ARN syndrome; Granulomatous uveitis; Visual loss and macular edema.

On The right eye visual acuity, was up to $20 / 20$, the slit lamp exam of this eye was unremarkable.

Angiography showed the presence of occlusive vasculitis and perimacular patchs of retinitis, associated with macular edema (Figure 1).

In view of the clinical aspect, an acute viral retinal necrosis was suspected, and an intra veinous antiviral-based treatment was started urgently with aciclovir at a dose of $10 \mathrm{mg} / \mathrm{kg} / 8 \mathrm{~h}$ followed $48 \mathrm{~h}$ later by a bolus of methylprednisolone at a dose of $500 \mathrm{mg}$ per day for 3 days.

Simultaneously a Polymerase chain reaction of the aqueous tap was done and it turn positive for HSV-1 and negative for HSV2, Varicella Zoster Virus (VZV) and Cytomegalovirus (CMV).the diagnosis of viral retinitis was made.

The evolution was marked by the disappearance of inflammatory reaction in the anterior chamber, a manifest decrease of vititis after 3 days of treatment. After 1 month of treatment, the visual acuity of the left eye is limited to 20/200, with a calm anterior segment and regression of peri-vascual sheath as well as macular edema (Figure 2).

The patient is currently on antiviral treatment taking oral prophylactic dose, oral corticosteroids in decreasing dose and IVT antiangiogenics due to the presence of an area of central ischemia
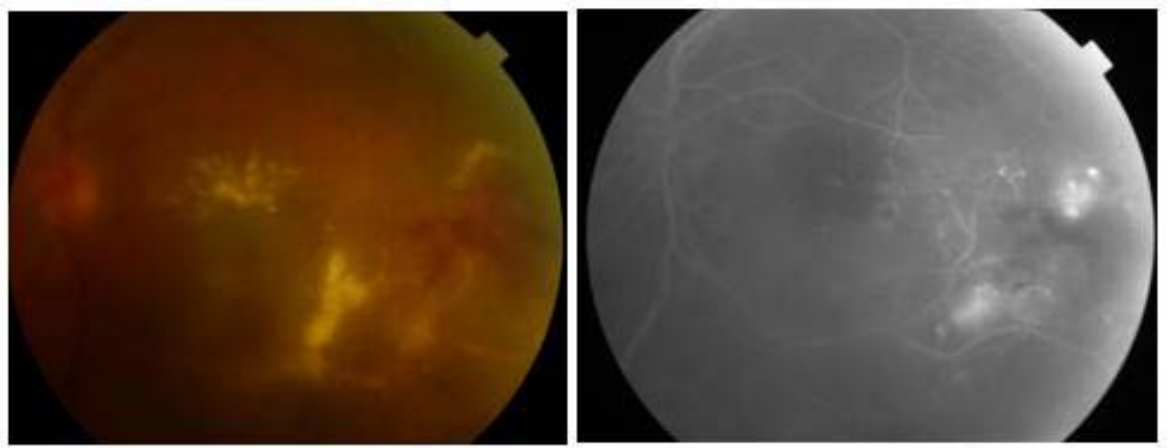

Figure 1: Acute retinal necrosis with severe vitritis and macular edema before treatment.

Austin J Clin Ophthalmol - Volume 8 Issue 2 - 2021 ISSN : 2381-9162 | www.austinpublishing group.com Khanaouchi et al. () All rights are reserved
Citation: Khanaouchi N, Khoyali A, Zerrouk R, Mouzarii Y, Reda K and Oubaaz A. Acute Retinal Necrosis a Potentially Visual Devastating Affection. Austin J Clin Ophthalmol. 2021; 8(2): 1118. 


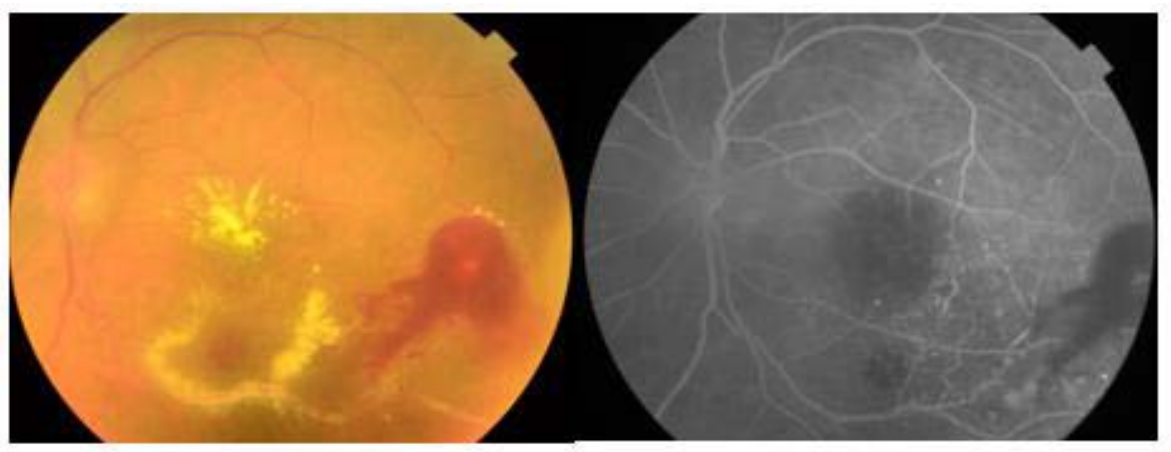

Figure 2: Manifest Improvement after antiviral treatment, disappearance of vitritis and regression of perivascular inflammation, although we notice the presence of perimacular ischemic zones with Micro-vascular angiopathy and neovascularization.

complicated by neovascularization not accessible to PRP.

\section{Discussion}

ARN syndrome is clinically defined by the presence of the classic triad associating full-thickness retinal necrosis of yellowish white appearance with peripheral location and well-limited edges, vasculitis and more or less severe vitritis. Other clinical manifestations may be indicative of this condition such as inflammation of the anterior segment complicated by ocular hypertonia, macular or papillary edema.

In decreasing order of the pathogens involved in ARN syndrome we find, varicella zoster virus in more than half of cases, followed by Herpes Simplex Virus (HSV), and then less frequently the EpsteinBarr virus. CMV-linked ARN syndrome remains very rare [1].

It affects both sexes with a slight male predominance, without preferential age, however two frequency peaks, were found, around 20 and 50 years old, related for the first to an attack by herpes simplex and for the second peak a varicella- zoster infection. And it is often found in immunocompetent individuals [1,2].

The mechanism of retinal infection is probably Reactivation in the central nervous system from a latent site of infection.

The main risk factor is a history of viral infections regardless of its location [3].

Several series have suggested a high prevalence of viral retinitis on a ground of genetic susceptibility. Holland and collegues, have demonstrated high prevalence of HLA-DQw7 antigen in patients with RNA syndrome than in the normal population, other genetic phenotypes one was highlighted such as Bw62, DR4 [1,4].

ARN syndrome is classically revealed by a rapidly progressive visual impairment, most often unilateral, associated with redness, eye pain and myodesopsia.

Examination of the anterior segment may reveal granulomarous anterior uveitis, hypertonia, and inflammatory reaction in the anterior chamber. Sometimes papillary and macular edema is associated to the retinal necrosis on fundus.

The diagnosis of this affection, is made on clinical presumption, no assessment should delay urgent treatment. Moreover, the detection of viral DNA by PCR technique, in aqueous humor or vitreous specimen is particularly interesting. Indeed PCR is very sensitive (80 to $96 \%$ ) and requires only a small amount of sampling [2].

Viral necrotizing retinitis can be confused with various ocular involvement including extensive toxoplasmic retinitis, syphilis, endophthalmitis of parasitic origin, ocular lymphoma and necrotizing retinitis in the context of behcet's disease, therefore an initial complete assessment is crucial without delaying the urgent therepeutique process.

The goal of treatment is to stop viral multiplication and the extension of the retinal necrosis as well as inflammation, it is based essentially on intra-veinous antiviral associated with antiinflammatory and antithrombotic treatment depending on virus isolated.

Acyclovir should be started at $10-13 \mathrm{mg} / \mathrm{kg}$ every 8 hours or $1500 \mathrm{mg} / \mathrm{m}^{2} /$ day intravenously (IV) for 5-10 days. This should be followed by acyclovir $800 \mathrm{mg}$ five times daily orally for 6 weeks to 3 months. Some studies suggest oral therapy at beginning such as Valacyclovir given at a dose of 1000-2000 mg orally every 8 hours. This has good bioavailability and avoids the need for intravenous access [5].

A long-term oral antiviral treatment is required to prevent recurrence. The visual prognostic is generally poor, severe complications may occur, such as retinal detachment, optic neuropathy and ischemic vasculopathy involving the macula can cause severe visual loss.

\section{Conclusion}

To conclude ARN is a rare but potentially visually threatening condition that can lead to blindness. First line therapy is intra-venous antiviral treatment, relayed by oral therapy to prevent recurrences. However, the cost of therapy is high and patient compliance can be a problem especially in developing countries, which make the prognosis of this affection more fatal in these cases.

\section{References}

1. Avrey Thau, Lauren Taney, Murtaza Adam. Acute Retinal Necrosis. American Academy of Ophthalmology.

2. Izambart C, Zur C, Fiandrino P, Tieulie N, Cassoux N \& Gastaud P. Prise en charge du syndrome de nécrose rétinienne aiguë compliqué d'œdème 
maculaire cystoïde. Journal Français d'Ophtalmologie. 2019; 32: 60.e1-60. e6.

3. Holland GN, Cornell PJ, Park MS, Barbetti B, Yuge J, Kreiger AE, et al. An association between acute retinal necrosis syndrome and HLA-DQw7 and phenotype Bw62, DR4. Am. J. Ophthalmol. 1989; 108: 370.
4. Jennifer I Lim, Koushik Tripathy, Lauren Taney, Murtaza Adam, Vinay A Shah and Vinay A. Shah. Acute retinal necrosis.

5. Bergstrom R, Tripathy K. Acute Retinal Necrosis. In: StatPearls. Treasure Island (FL): StatPearls Publishing. 2021. 